\title{
A Review for Aircraft Landing Problem
}

\author{
Li Zipeng ${ }^{1, a}$ and Wang Yanyang ${ }^{1, b}$ \\ ${ }^{1}$ School of Aeronautic Science and Engineering, Beihang University, Beijing, China
}

\begin{abstract}
Substantial increase in the amount of civil aviation traffic and other factors have led to frequent congestion and adverse effects in terminal area. Therefore, the landing sequence of aircrafts need to be managed so as to reduce adverse effects. This type of problem is called Aircraft Landing Problem (ALP), and is an optimization problem. In this paper, the current situation of ALP research is analysed. The specific process of landing is described, and the existing research problems are classified. At the same time, on the aspect of optimization, this paper discusses the optimization processing of ALP, including the establishment of optimization model and the design of optimization algorithm. In the last, the future research trend of ALP is proposed as conclusions.
\end{abstract}

\section{Introduction}

With the growth of civil aviation transportation, numerous aircrafts need to land within a short time. Due to shortage of aviation resources, flights delay is caused, which resulting in reduced efficiency, economic losses and other adverse effects.

However, expanding the airport runway facility simply is limited by various factors, so the need for reasonable management of aircrafts to reduce the adverse effects increases. Such problem is called Aircraft Landing Problem (ALP), which is an important issue in air traffic control.

\section{Description of ALP}

The landing process is described as follows. The approaching aircrafts flew to the terminal area from different directions. The airspace of the terminal area is divided by the initial and the final scheduling boundaries, between which is the scheduling window. If an aircraft enters this area, the algorithm accepts the following inputs: the specific time aircraft enters, the estimated time of arrival (ETA) which is predicted by the traffic control tool of ATC, the weight level of the aircraft and so on. After calculating, the scheduling algorithm allocates the runway and arrange scheduled time of arrival (STA) for aircrafts. After scheduling, aircrafts reach the converging point and enter the frozen area in which landing sequence is not changed unless exceptional cases.

ALP is an optimization problem, belonging to the NP-hard problem [1]. The model of ALP can be divided into basic or detailed models [2]. The basic model only considers runways as constraint of the airport capacity in the terminal area, and it can be abstracted as single or parallel machine scheduling

\footnotetext{
${ }^{a}$ Li Zipeng : lzplzp37@buaa.edu.cn

${ }^{\mathrm{b}}$ Wang Yanyang : Wangyanyang@buaa.edu.cn
} 
problem in the machine scheduling problem [3]. The detailed model takes runways and other related terminal resources into account, which can be abstracted as a job shop scheduling problem.

\section{Classification of ALP}

Using the basic model, ALP can be divided into single and multiple runway problems. Using detailed models, considering the airport resources, ALP can be transferred into corresponding problems in multi-airport environment.

\subsection{Problems for single or multiple runways}

The airport can be divided into single and multiple runways airport according to the number of runways. Single runway problem (SRP) means that all aircrafts are scheduled to land on the same runway. Multi-runway problem (MRP) need to do runway assignment as well as sequencing. In essence, SRP can be abstracted as a single server queue in queuing theory, and MRP can be abstracted as multiple servers queue, including sequencing and distribution.

In China, most airports have one runway, so the study of SRP still makes sense. Its trend is to improve the accuracy and efficiency of the solution constantly, as well as to be the basis of research in multi-runway problem.

The current research mainly focuses on MRP. As a result of the emergence and continuous improvement of heuristic algorithms, the scale of problem solved grows, and precision and efficiency of calculation are gradually improved.

Multi-runway airports have different runway configurations, which will affect the airport capacity, and the current study is mainly based on parallel runways configuration. In China, most multi-runway airports use parallel runways configuration, but there are still some airports using other configurations like new airport in Chengdu, using two parallel runways and a lateral runway, and it will have an impact in wake interval.

The parallel runways also have different modes of operation, including: independent or relative parallel instrument approach and so on. In independent parallel instrument approach mode, the various runways are independent. In the relevant parallel instrument approach mode, aircrafts landing on the adjacent runways need to meet a certain radar interval. The current research is mainly based on independent parallel instrument approach mode.

\subsection{Problems for multi-airport}

Multi-airport terminal area refers to the terminal area including two or more airports, and arrivals and departures of these airports interrelate.

In addition to the multi-runway sequencing at the airports, the multi-airport problem also includes sequencing in the entry point of terminal area. According to the basic principle of queuing theory, if entry points, airports, runways and other key nodes can be considered as a multi-server system, the multi-airport problem can be abstracted as multi-server parallel queuing events.

The study of multi-airport problem starts late, and many researches are incomplete. For example, someone considering the space resources of entry point and runways simply, combined the queuing theory and designed algorithm for ALP without considering the intersections in the airspace.

However, the current study of multi-airport, in addition to the proposed shortages like single optimization goal, ignoring the safety interval of cross-route, also lose sight of the number of runways of different airports, complex runway configuration and the impact of takeoff flow.

\section{Method}


In ALP, according to the model, allocation and sequence of landing should be provided ensuring constraints and specific need of different stakeholders. Based on above requirement, the key elements of ALP are extracted and the conceptual model is constructed as Fig.1.

The ALP covers the establishment of optimization model and the design of algorithm. The optimization model is composed of decision variables, optimization goals and constraints.

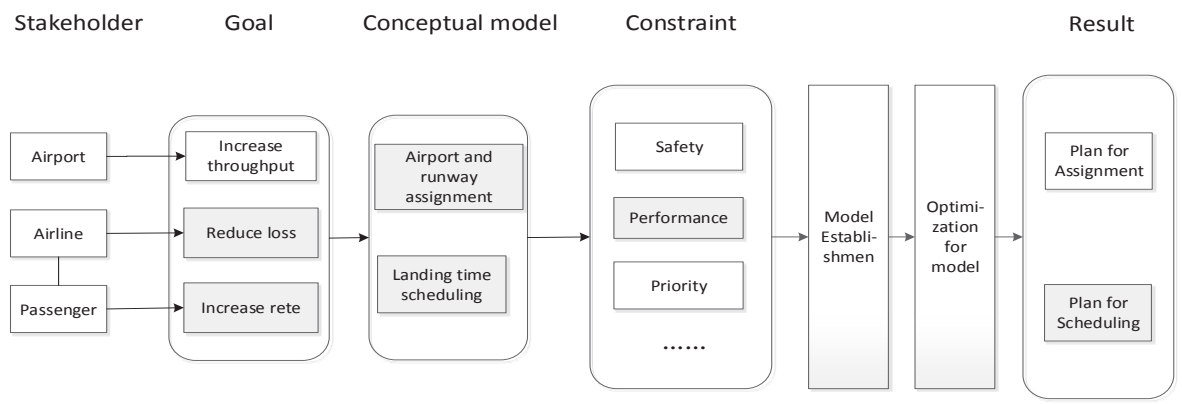

Figure 1. Conceptual model of ALP

\subsection{Optimization goals}

ALP involves aspects of benefits. ATC concerns about the safety; airline concerns about its economic losses; airports concerned about the delay rate; passengers concerned about the flight punctuality rate. Different angles result in the different optimization goals.

The current optimization goal can be divided into single and multi-objective goal. Common expression of single goal are time minimization and cost minimization.

Time minimization focuses on the ability of solving terminal congestion, mainly with forms of minimal delay or landing completion time. Minimal delay is the most common goal for ALP, which represents the shortest waiting time in the air and achieves maximal airport throughput. Minimal landing completion time is suitable for short-term terminal congestion.

Cost minimization focuses on airport operating costs and airline benefits. The cost is sometimes expressed as a linear or square relationship with time, which cannot adequately reflect the actual complex situation, and it can be divided into various expenses according to considerations. The study at Delft University divides expenses into passenger spending subsidies, passenger inconvenience, fuel consumption, etc. Similarly, someone divides expenses into operating expenses of resource path, flight delays expenses and flight cancellation expenses.

Other angles can also be chosen as goals. For example, Gini coefficient is used as optimization goal to ensure equitable distribution of resources between airlines.

As the number of concerns increases, the optimization goal in recent years has evolved into multiple to balance requirements of various aspects, and ALP is transformed into a multi-objective optimization problem (MOO).

The common solving of MOO problem is to change it into a mature single-objective optimization problem, such as setting the weight coefficient for different optimization goals. However, it is difficult to evaluate the solution objectively.

In the multi-airport environment, multiple optimization goals are proposed and the goal of fairness of public airspace resources use is added to reduce large delays of certain airport. Non-dominated sorting genetic algorithm with elite strategy is used to solve MOO and obtain the Pareto optimal solution.

\subsection{Constraints}

ALP belongs to multi-constraint problems. Constraints can be divided into multiple types, including safety constraints which maintain flight safety, performance limits which characterize the performance of aircrafts to avoid the results beyond the actual range, priority constraints which permit certain 
flights to be prioritized, fairness constraints which ensure the fairness of sequencing and cost constraints which can reduce loss to some extent.

Common typical constraints are as follow.

First one is the minimum interval constraints which belongs to safety constraints. It mainly refers to the horizontal interval in space, and can be divided into wake or radar interval constraint according to different causes.

Two successive aircrafts can reduce the threat of wake maintaining certain distance, which introduced the wake interval constraints. The minimal distance is real-time calculated based on speed and weight. In order to simplify the problem, ICAO specifies the minimum wake space interval between different weight types of aircrafts, and the distance interval is converted into the time interval in order to meet the time-based needs of ATC. The time-base process may bring some defects, such as lack of accuracy, but nowadays, the standard is widely used when dealing with ALP.

Radar interval constraints are unique to MRP. When the mode of relative parallel instrument approach is used, two successive aircraft landing on different runways need to meet the lateral radar interval.

Second one is the time window constraint belonging to performance constraints. Each aircraft has earliest and latest landing time, which are related to its flight performance and its position. Aircrafts will land during the time window.

Third one is runway capacity constraints belonging to safety constraints. It means that each runway can only serve an aircraft at one time.

Fourth one is the maximal position shift constraint (MPS) and the relative position shift constraint (RPS). They refer to the range of aircrafts position shift relative to the original sequence and the last sequence. These constraints limit the range of movements to a certain extent, which can ensure that aircrafts will not be led to fuel exhaustion or affecting landing order, which relates to safety and performance. This constraint can also avoid large delay, ensuring the fairness of the sequencing.

Among them, the minimum wake interval constraint is vital, which will affect runway capacity at peak hours, and runway capacity constraints must also be taken into account. Other constraints are used to improve the landing requirements better.

\subsection{Algorithms}

Since ALP is an NP-hard problem, listing all possibilities will introduce large calculations, and is not suitable for practical use. Currently, common algorithms solving ALP are divided into three categories: FCFS algorithm, exact algorithm and heuristic or meta-heuristic algorithm.

Due to the lack of effective accessorial decision-making tools, FCFS is a method currently used in the manual scheduling environment, especially for single runway problems. FCFS sequences the aircrafts according to their ETAs. It is simple and quick, and can obtain good solution in the uncrowded terminal area. However, it may bring greater delay in rush hours and has been unable to meet the current needs of air traffic management.

Subsequently, FCFS is improved to time advance algorithm (TA) and delay exchange algorithm (DE). TA accelerates the first flight to reduce the sum of delay time without changing order, and DE only advances urgent flights. These methods do not contain idea of optimization, and not practical when the number of flights increased.

Exact algorithms are used to deal with linear programming problems, including branch and bound algorithm, column-and-row generation [4], branch-and-price algorithm [5], dynamic programming algorithm and so on. It always builds 0-1 mixed model to solve ALP [6]. However, such algorithms cannot even solve the problem of medium size, which makes people begin to consider algorithms of new type [7].

As a result, heuristic algorithms are gradually applied to ALP. They are usually designed by imitating some phenomena or processes in nature, having the advantage of high parallelism, and can deal with nonlinear complex model. Although the optimal solution cannot be guaranteed, the approximate solution can be obtained within reasonable time, and under reasonable computing 
resource conditions, the solution will be of high quality. Common heuristic algorithms include ant colony algorithm, genetic algorithm [8], particle swarm algorithm [9], cellular automata algorithm, bat algorithm and so on. They can handle many optimization problems. However, they all have their own shortcomings, and need to be improved or used in combination to make the problems better solved.

For example, classical genetic algorithm (GA) cannot search the global optimal solution unless the optimal individuals of each generation are preserved. With the complexity of problems, the defects of classical GA are emerging. Therefore, it is improved to maintain the diversity of species, or be combined with other algorithms to improve the performance, such as adaptive annealing GA. GA used by ALP is also modified with the development of GA. A non-dominated sorting genetic algorithm is designed with elite strategy to ensure the optimality and abundance of population so that efficiency of calculations can be improved. Someone used the adaptive evaluation vector to guide the search of the results for the short-term ALP and adopt the multi-objective genetic algorithm based on inequality. These improvements make the algorithms more advantageous.

Other heuristic algorithms can also have better performance through a certain improvement. In the literature [10], the improved strategy is proposed for the traditional particle swarm algorithm which is easy to fall into local optimum and has shortcomings in solving combinatorial optimization problem.

The improved algorithm generates the initial particle swarm through tabu search strategy to meet the multiple constraints of ALP, introduces the operations of cross and mutation in GA to enhance the information exchange ability between particles, redefines the inertia weight and acceleration factor to enhance the diversity and convergence of particles, adding the evolutionary crossover operator to enhance the local search ability. Through comparison, it is proved that the abilities of local and global search are enhanced.

Combining heuristic algorithms can improve the accuracy or efficiency to a certain extent. For example, immune concentration adjustment mechanism is added and tabu search algorithm is combined with particle swarm algorithm, which avoids being easy to fall into the local optimum and improves the efficiency. Artificial fish swarm algorithm and particle swarm algorithm are also combined together. Artificial fish swarm algorithm is used for global search, and particle swarm optimization algorithm is used to find the local optimum, which overcomes the shortcomings of slow convergence in artificial fish swarm algorithm and of being easy to fall into the local optimum in the particle swarm algorithm, thus improving the search speed and convergence accuracy.

\subsection{Static and dynamic situation}

Dynamic and static situations involve whether there are new aircrafts flying into terminal and whether results are to be modified in real time in the sequencing process.

From static to dynamic, there are three common ways, one is to change the optimization model, such as adding reordering cost into the goals. When aircrafts need to be rescheduled, Beasley used shift penalty function to evaluate the expense of changing, and control shifts within a certain range. Second, according to the definition of the dynamic situation, the new flight joining the queue can be regarded as a trigger event model, and flights should be reordered after trigger happens. Third, receding horizon control (RHC) strategy [11] can be added to achieve dynamic. For example, B.S.Girish applied the hybrid particle swarm algorithm in the framework of a receding horizon, and Marcella Samà combined the receding horizon algorithm with the branch and bound algorithm to achieve dynamic and improve the efficiency of computing. The frequency of new flights joining the queue is not regular, so the sequencing frequency is also irregular to follow, and use of RHC in a fixed frequency to implement the algorithm can meet its dynamic needs to a certain extent.

\section{Tendency}

Many runways use a mixed mode of operation, so it is necessary to consider takeoff aircrafts on the same runway to avoid accidents [12]. However, most studies ignored departing flights in the terminal area. Some studies take departing flights into account without considering the trigonometric inequality 
of interval. Ghoniem A [13] solved this problem by introducing new simple interval constraints in two aircrafts of identical weight level and operation to ensure the inequality. However, the above method can get better results for arrivals, and departures do not get a good arrangement. Therefore, the need to consider arrivals and departures together is increased so that the appropriate solution for both sides can be obtained.

ALP is usually more complicated. Runways have different modes of operation, and they may be mixed operated or can only be used for take-off or landing [14], which is seldom taken into account in research. In future studies, similar complex situations should be considered.

In addition, optimization goals need to be improved. Minimization of time or economic losses does not adequately meet actual needs. In practice, the delay or economic loss of different aircrafts cannot be treated equally, and the weight factor need to be considered.

At the same time, the aircraft may have the problem of insufficient fuel due to conversion problems. However, the existing research does not take these factors into account.

\section{Conclusions}

ALP is gradually converted into a dynamic, multi-airport, multi-runway problem. Multi-runway, multi-airport is the future trend of construction. Meanwhile, hub airports have growing traffic and busy terminal area, which need to be considered as a dynamic problem.

Optimization goals are changed gradually from single to multiple [15]. They are no more simply time delay or economic loss. Fuel coefficient and other aspects of comprehensive consideration should also be added. Constraints will become more comprehensive, and multi-runway problem make some new constraints need to be introduced. With the frequent use of heuristic algorithms, the improvement and combination of algorithms has been ongoing, and the direction of which is always to enhance its accuracy and efficiency.

\section{Acknowledgement}

This research was supported by the National Science and Technology Major Project (Grant No. 2016ZX04004006).

\section{References}

1. B. S. Girish, Applied Soft Computing 44 200-21 (2016)

2. M. Samà, A. D'Ariano, D. Pacciarelli, Transportation Research Part E: Logistics and Transportation Review 60 140-55 (2013)

3. S. Vadlamani, S. Hosseini, J. of Air Transport Management 40 144-8 (2014)

4. Maher S J, Transportation Science (2015)

5. A. Ghoniem, F. Farhadi, M. Reihaneh, Eur. J. of Operational Research 246(1) 34-43 (2015)

6. A. Jacquillat, A. R. Odoni, Operations Research 63(6) 1390-410 (2015)

7. A. Lieder, D. Briskorn, R. Stolletz, Eur. J. of Operational Research 243(1) 61-69 (2015)

8. H. Zhou, X. Jiang, Mathematical Problems in Engineering (2015)

9. A. A. A. Esmin, R. A. Coelho, S. Matwin, Artificial Intelligence Review 44(1) 23-45 (2015)

10. M. Yingjun, S. Xiaona, Z. Dongfang, Application Research of Computers 7030 (2015)

11. F. Furini, M. P. Kidd, C. A. Persiani, et al, 18(5) 435-447 (2015)

12. G. Hancerliogullari, G. Rabadi, A. H. Al-Salem, J. of Air Transport Management 32 39-48 (2013)

13. A. Ghoniem, H. D. Sherali, H. Baik, INFORMS Journal on Computing 26(3) 514-30 (2014)

14. A. Lieder A, R. Stolletz, Transportation Research Part E: Logistics and Transportation Review 88 167-88 (2016)

15. W. Malik, Y. C. Jung, 16th AIAA Aviation Technology, Integration, and Operations Conf. 4072 (2016) 